\title{
Circulating miR-221 and miR-222 as Potential Biomarkers for Screening of Breast Cancer
}

\author{
Jungho Kim ${ }^{1,2, \S, *}$, Sehee $\mathrm{Oh}^{1,3, \$, * *}$, Sunyoung Park ${ }^{1, *}$, Sungwoo Ahn ${ }^{1, *}$, Yeonim Choi ${ }^{4, * * *}$, \\ Geehyuk Kim ${ }^{1, *}$, Seung II Kim ${ }^{5, * * *}$ and Hyeyoung Lee ${ }^{1, \dagger, * * *}$ \\ ${ }^{I}$ Department of Biomedical Laboratory Science, College of Health Sciences, \\ Yonsei University, Wonju, Gangwon 26493, Korea \\ ${ }^{2}$ Department of Microbiology, Institute of Immunology and Immunological Disease, \\ College of Medicine, Yonsei University, Seoul 03722, Korea \\ ${ }^{3}$ DNA Analysis Section, Division of Forensic Medicine, Busan Institute, \\ National Forensic Service, Yangsan 50612, Korea \\ ${ }^{4}$ Department of Biomedical Laboratory Science, Songho College, Hoengseong, Gangwon 25242, Korea \\ ${ }^{5}$ Department of Surgery, College of Medicine, Yonsei University, Seoul 03722, Korea
}

\begin{abstract}
Breast cancer is the second most common cancer in women with approximately 522,000 deaths annually worldwide. microRNAs have recently been studied as potential biomarkers that regulate gene expression and are involved in tumorigenesis. Here we evaluated circulating miR-221 and miR-222 as potential biomarkers for breast cancer by quantitative reverse transcription PCR using blood plasma of 30 healthy controls and 30 breast cancer patients. The TNM stage on circulating miR-221 and miR-222 was also investigated. Circulating miR-221 and miR-222 were significantly up-regulated in breast cancer patients compared to those in healthy controls $(P<0.0022$ and $P=0.0058$, respectively). Furthermore, the relative expression level of circulating miR-221 in patients with stage III breast cancer was higher than in those with stage I and II. Taken together, we have shown circulating miR-221 and miR-222 could be useful biomarkers for the screening of breast cancer patients.
\end{abstract}

Key Words: Breast cancer, Screening, miR-221, miR-222, Biomarker

Breast cancer is the most frequently occurring disease in females and the second leading cause of death worldwide (Siegel et al., 2018). According to the World Health Organization (WHO), approximately 522,000 women die each year from breast cancer (Ferlay et al., 2015). Current methods used to diagnose breast cancer such as mammography, computed tomography (CT), and magnetic resonance imaging
(MRI) have been hindered by the cost and expertise needed. In addition, tumor serum markers such as carbohydrate antigen 15-3 (CA15-3) and carcinoembryonic antigen (CEA) are nonspecific and have limited sensitivity and specificity (Duffy, 1999; Ng et al., 2014). Therefore, it is necessary to discover new non-invasive biomarkers for the diagnosis of breast cancer.

Received: June 13, 2019 / Revised: June 23, 2019 / Accepted: June 25, 2019

*Post-Doctor, ${ }^{* *}$ Researcher, ${ }^{* * *}$ Professor.

$\S$ Jungho Kim and Sehee Oh contributed equally.

'Corresponding author: Hyeyoung Lee. Department of Biomedical Laboratory Science, College of Health Sciences, Yonsei University, 1 Yonseidae-gil, Wonju-si, Gangwon-do 26493, Korea.

Tel: +82-33-760-2740, Fax: +82-33-760-2561, e-mail: hyelee@ yonsei.ac.kr

(C) The Korean Society for Biomedical Laboratory Sciences. All rights reserved.

(c) This is an Open Access article distributed under the terms of the Creative Commons Attribution Non-Commercial License (http://creativecommons.org/licenses/by-nc/3.0/) which permits unrestricted non-commercial use, distribution, and reproduction in any medium, provided the original work is properly cited. 
microRNAs are small non-coding RNAs consisting of $18 \sim 25$ nucleotides that play a role in regulating gene expression by binding the target messenger RNAs (mRNAs). Numerous recent studies have reported that microRNAs, which are involved in tumorigenesis, are a potential diagnosis, prognosis and therapeutic biomarker in various cancers (Achkar et al, 2016). miR-221 and miR-222 are located on the $\mathrm{X}$ chromosome with the same base sequence. Researchers have shown that miR-221 and miR-222 are over-expressed in breast cancer cells and function as oncomiRNAs (Garofalo et al., 2012). Previous studies also show that miR-221/222 promotes breast cancer cell proliferation, migration, and invasion by targeting PTEN, a tumor suppressor gene ( $\mathrm{Li}$ et al., 2016). However, the clinical relevance of miR-221/222 in plasma is still poorly investigated. In this study, circulating miR-221 and miR-222 as potential biomarkers for breast cancer were investigated using the blood plasma of 30 healthy controls and 30 breast cancer patients.

A total of 30 blood samples were obtained from breast cancer patients who had been diagnosed with breast cancer at the Yonsei University Severance Hospital, Seoul, Republic of Korea from 2000 to 2015 . For the healthy controls, a total of 30 blood samples were obtained from healthy donors who had never been diagnosed with breast cancer. All patients and healthy volunteers provided written consent and the study was approved by the institutional ethics committee at Yonsei University Severance Hospital (approval no. 4-2011-0011) and Yonsei University at Wonju (approval no. 1041849201603-BR-010-04).

For separation of plasma, the whole blood samples were centrifuged for $15 \mathrm{~min}$ at $600 \times \mathrm{g}$ and stored at $-80^{\circ} \mathrm{C}$ in the deep freezer until use. For extraction of small RNAs from the plasma of the study population, the NucleoSpin ${ }^{\circledR}$ miRNA Plasma kit (Macherey-Nagel, Düren, Germany) was used according to the manufacturer's protocol.

miRNA expression was quantified by using the TaqMan ${ }^{\circledR}$ small RNA assays (Applied Biosystems by Life Technol ogies) with miRNA specific primers (miR-221 and miR-222) to measure the cycle threshold $\left(\mathrm{C}_{\mathrm{T}}\right)$ - the number of PCR cycles in which fluorescence exceeds the background fluorescence value. The samples were repeated twice and the data were analyzed using the comparative $\Delta \mathrm{C}_{\mathrm{T}}$ method $\left(2-{ }_{\mathrm{T}}{ }_{\mathrm{T}}\right)$
Table 1. Clinical characteristics of breast cancer patients

\begin{tabular}{lrr}
\hline \hline \multicolumn{1}{c}{ Variable } & Cases & $\%$ \\
\hline Age & & \\
$\quad<50$ 's & 8 & 26.7 \\
$\geq 50$ 's & 21 & 70.0 \\
Unknown & 1 & 3.3 \\
TMN stage & & \\
I & 10 & 33.3 \\
II & 10 & 33.3 \\
III & 10 & 33.3 \\
\hline
\end{tabular}

with miR-16 as an endogenous control (Pfaffl et al., 2002).

We assessed the relative expression level of circulating miR-221 and 222 in 30 healthy control and 30 breast cancer patients. The characteristics of the patients are shown in Table 1. The age of the breast cancer patients ranged from 31 to 76 years old (mean $\pm \mathrm{SD}, 55.03 \pm 11.31$ years; $95 \mathrm{CI}$, 50.73 59.34). Of 30 breast cancer patients, 10 (33.3\%) were in stage I, followed by 10 (33.3\%) in stage II, and 10 (33.3\%) in stage III. The expression levels of circulating miR-221 and miR-222 in breast cancer patients were significantly higher than those in the healthy controls $(P<0.0022$ and $P$ $=0.0058$, respectively) (Fig. 1). The mean expression levels of circulating miR-221 and miR-222 were 0.2569 (range, 0.0024 to 2.6240 ) and 0.0562 (range 0.0003 to 0.3530 ) in breast cancer patients and 0.0895 (range, 0.0011 to 0.3184 ) and 0.0230 (range 0.0027 to 0.0641 ) in healthy controls. The area under the ROC curve (AUC) of circulating miR-221 and miR-222 was 0.7267 (95\% CI, 0.5983 0.8551, $P=$ $0.0025)$ and 0.7056 (95\% CI, 0.5683 0.8428, $P=0.0062$ ), respectively.

Subsequently, the expression levels of circulating miR221 and miR-222 in different stages were analyzed. There was a statistically significant difference between the miR221 expression level of stage II and stage III and that of the healthy controls $(P=0.0279$ for stage II and $P=0.0163$ for stage III) (Fig. 2A). For miR-222, there also was a significant difference between stage II and the healthy controls $(P=$ 0.0084) (Fig. 2B).

Many recent studies have reported blood-based biomarkers for diagnosing breast cancer, such as circulating tumor cells 
A

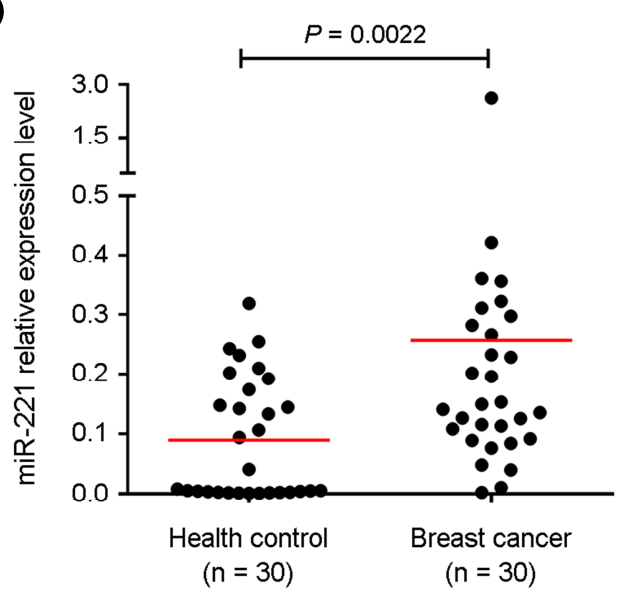

ROC of miR-221

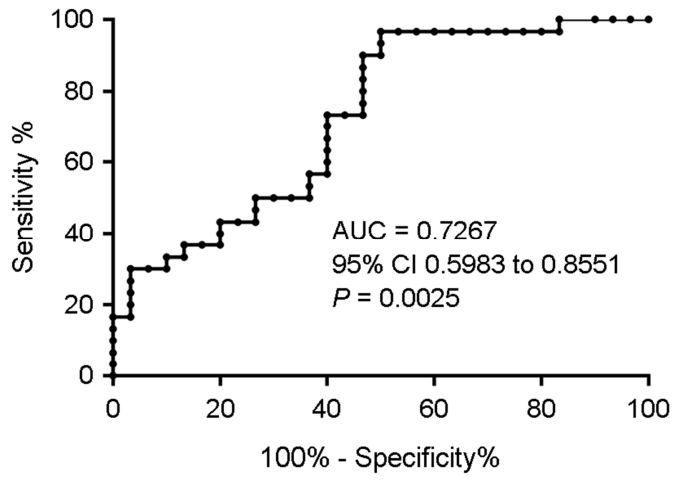

B

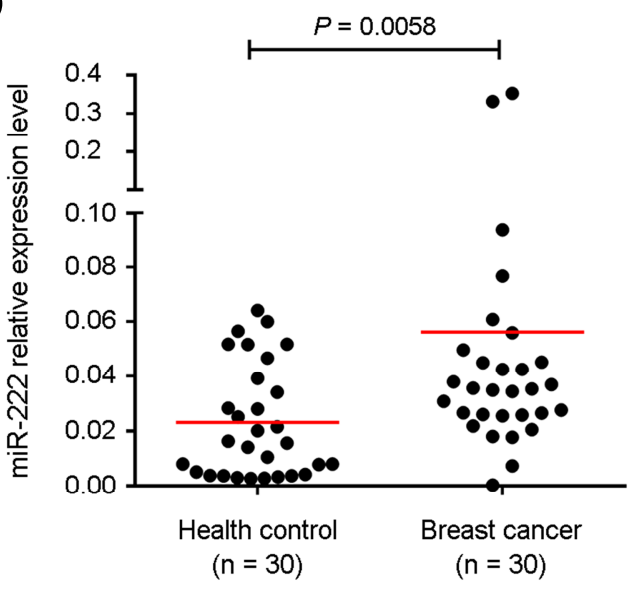

ROC of miR-222

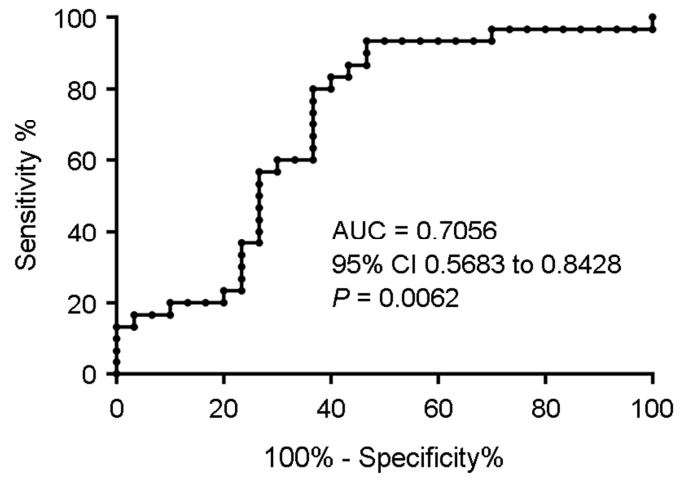

Fig. 1. Circulating miR-221, and miR-222 expression level in plasma of breast cancer patients and healthy controls. The relative expression of miR-221 (A), and miR-222 (B) in plasma of breast cancer patients and healthy controls was analyzed by RT-qPCR.

A

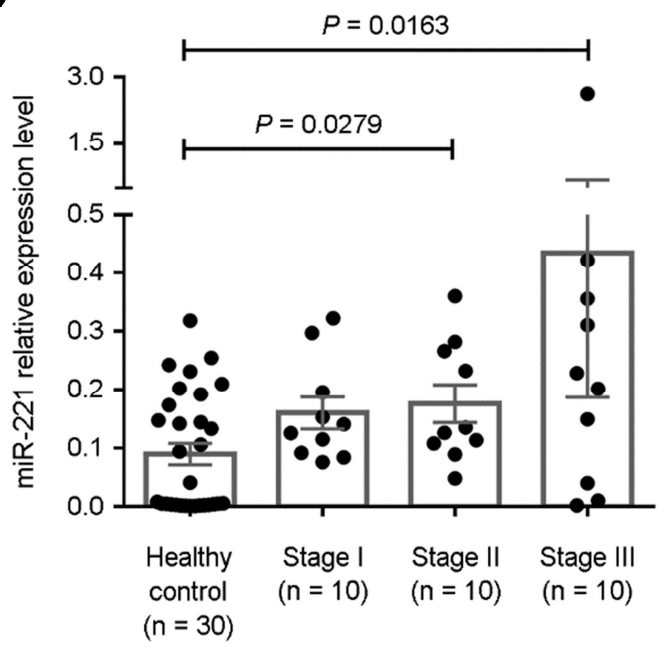

B

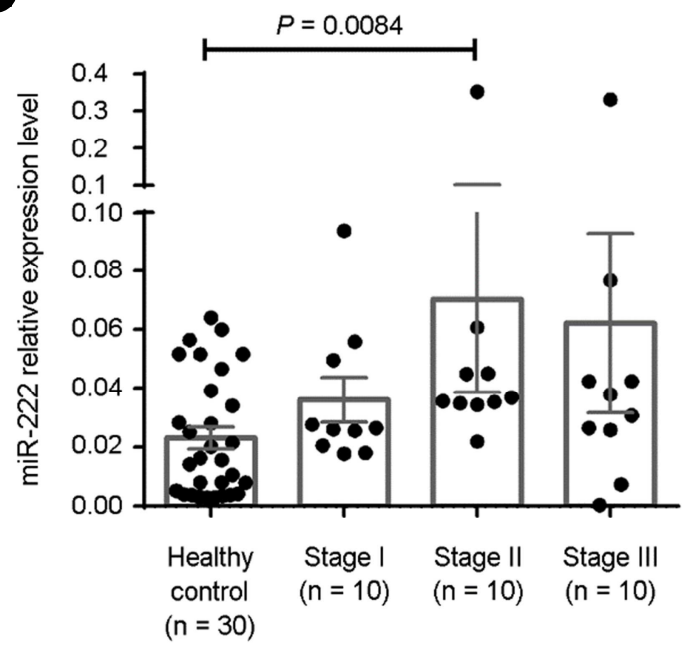

Fig. 2. Circulating miR-221, and miR-222 expression level according to TNM stage. The miR-221 (A) and miR-222 (B) expression levels were analyzed at different breast cancer stages. 
(CTCs), circulating tumor DNA (ctDNA), exosomes, and microRNAs. Circulating miRNAs have many advantages as biomarkers, such as stability during $\mathrm{pH}$ changes, freezethaw cycles, and extended storage (Mitchell et al., 2008; Yu et al., 2011). In this study, circulating miR-221 and miR-222 were potential biomarkers showing 2.87-fold and 2.44-fold higher expression for breast cancer patients compared with healthy controls, respectively.

In this study, some healthy controls were shown high expression of miR-221 and miR-222 or some patients with stage III were shown low expression of miR-221 and miR222. The reason for discrepancies of miR-221 and miR-222 expression in healthy controls and breast cancer patients was that microRNAs regulate multi-genes rather than a single gene. For example, miR-221/222 was associated with oncogenic process regulating tumor suppressor gene PTEN (Li et al., 2016). Also, miR-221/222 was associated with inflammatory immune response regulating peroxisome proliferatoractivated receptor- $\gamma$ coactivator- $1 \alpha$ (Song et al., 2017).

There are limitations to our study. First, a small number of samples were included. Therefore, it is necessary to conduct further research using a larger number of samples from breast cancer patients at various stages to demonstrate the utility of circulating miR-221 and miR-222 for breast cancer screening and diagnosis. Second, we only included breast cancer patients with cancer stage I-III. Further study with stage IV patients is needed to investigate the expression of circulating miR-221 and miR-222 with breast cancer stage IV patients.

In conclusion, expression levels of miR-221 and miR-222 in plasma were significantly higher for breast cancer patients than for healthy controls. Notably, miR-221 in patients with stage III breast cancer was higher than in those with stage I and II. Taken together, circulating miR-221 and miR-222 can serve as potential non-invasive biomarkers for breast cancer screening and diagnosis.

\section{ACKNOWLEDGEMENT}

None.

\section{CONFLICT OF INTEREST}

The authors have no conflicts of interest with regards to this study.

\section{REFERENCES}

Achkar NP, Cambiagno DA, Manavella, PA. miRNA biogenesis: A dynamic pathway. Trends Plant Sci. 2016. 21: 1034-1044.

Duffy MJ. CA 15-3 and related mucins as circulating markers in breast cancer. Annals of Clinical Biochemistry. 1999. 36: 579 $-586$

Ferlay J, Soerjomataram I, Dikshit R, Eser S, Mathers C, Rebelo M, Parkin DM, Forman D, Bray F. Cancer incidence and mortality worldwide: Sources, methods and major patterns in GLOBOCAN 2012. Int J Cancer. 2015. 136: E359-386.

Garofalo M, Quintavalle C, Romano G, Croce CM, Condorelli G. miR221/222 in cancer: Their role in tumor progression and response to therapy. Current Molecular Medicine. 2012. 12: 27-33.

Li B, Lu Y, Wang H, Han X, Mao J, Li J,Yu L,Wang B, Fan S, Yu X, Song B. miR-221/222 enhance the tumorigenicity of human breast cancer stem cells via modulation of PTEN/Akt pathway. Biomed Pharmacother. 2016. 79: 93-101.

Mitchell PS, Parkin RK, Kroh EM, Fritz BR, Wyman SK, Pogosova-Agadjanyan EL, Peterson A, Noteboom J, O'Briant KC, Allen A, Lin DW, Urban N, Drescher CW, Knudsen BS, Stirewalt DL, Gentleman R, Vessella RL, Nelson PS, Martin DB, Tewari M. Circulating microRNAs as stable blood-based markers for cancer detection. Proceedings of the National Academy of Sciences of the United States of America. 2008. 105: 10513-10518.

Ng EKO, Li R, Shin VY, Siu JM, Ma ESK, Kwong A. MicroRNA143 is downregulated in breast cancer and regulates DNA methyltransferases $3 \mathrm{~A}$ in breast cancer cells. Tumor Biology. 2014. 35: 2591-2598.

Pfaffl MW, Horgan GW, Dempfle L. Relative expression software tool (REST) for group-wise comparison and statistical analysis of relative expression results in real-time PCR. Nucleic Acids Res. 2002. 30: e36.

Siegel RL, Miller KD, Jemal A. Cancer statistics, 2018. CA Cancer J Clin. 2018. 68: 7-30.

Song JL, Ouyang YM, Che JY, Li XM, Zhao Y, Yang KJ, Zhao XT, Chen YH, Fan CY, Yuan WE. Potential Value of miR-221/222 as diagnostic, prognostic, and therapeutic biomarkers for diseases. Frontiers in immunology. 2017. 8.

Yu DC, Li QG, Ding XW, Ding YT. Circulating microRNAs: potential biomarkers for cancer. Int J Mol Sci. 2011. 12: 2055 $-2063$. 
https://doi.org/10.15616/BSL.2019.25.2.185

Cite this article as: Kim J, Oh S, Park S, Ahn S, Choi Y, Kim G, Kim SI, Lee H. Circulating miR-221 and miR222 as Potential Biomarkers for Screening of Breast Cancer. Biomedical Science Letters. 2019. 25: 185-189. 